\title{
Métodos innovadores y enfoques de aprendizaje en estudiantes universitarios
}

\author{
Bernardo Gargallo López ${ }^{\mathrm{a}}$ Eloïna García Félix ${ }^{\mathrm{b}}$, Isabel Morera Bertomeu ${ }^{\mathrm{c}}$ y \\ Amparo Benavent Garcés ${ }^{d}$ \\ a Departamento de Teoría de la Educación, Universidad de Valencia, bernardo.gargallo@uv.es, \\ bDepartamento de Métodos de Investigación y Diagnóstico en Educación, Universidad de Valencia, \\ algarcia@ice.upv.es, c Grupo GIIMA. Departamento de Química. Universitat Politècnica de \\ València, imorera@upvnet.upv.es, y dDepartamento de Enfermería, Universidad de Valencia, \\ Amparo.Benavent@uv.es
}

\begin{abstract}
The aim of this work was to assess whether the use of innovative methods, learner-centered, improved the learning approaches of university students. The study was developed with two students groups of 1st year $(N=112)$, from the degree of Pedagogy at the University of Valencia. A preexperimental pretest-posttest design was used with measures by means of the R-SPQ-2 questionnaire (Biggs, Kember and Leung, 2001). Both teachers agreed on the methodology, which included expository teaching, questions, classroom discussion, classroom practices, case study, cooperative work, development of a research work and presentation in the classroom, and an evaluation system that made use of training procedures that returned feedback to students (two portfolios, assessment of oral presentations, rubrics, co-evaluation, self-assessment and written tests.). Statistically significant improvements were found in scores of deep approach to learning, both in the overall score (deep approach) as in two dimensions scores, assessed by questionnaire (motives and strategies). No statistically significant differences were found in scores of surface approach, although they decreased.
\end{abstract}

Keywords: innovative methods, learner-centred teaching, learning approaches, university students 
Métodos innovadores y enfoques de aprendizaje en estudiantes universitarios

\begin{abstract}
Resumen
Se buscaba valorar si la utilización de métodos innovadores, centrados en el aprendizaje, mejoraba los enfoques de aprendizaje de los estudiantes universitarios. El estudio se realizó con dos grupos de estudiantes de $1^{\circ}$ curso $(N=112)$, del grado de Pedagogía de la Universidad de Valencia. Usamos un diseño preexperimental pretest-postest utilizando el cuestionario R-SPQ-2 (Biggs, Kember y Leung, 2001). Los dos profesores consensuaron la metodología, que comportaba enseñanza expositiva reducida, preguntas, discusión en clase, clases prácticas, estudio de casos, trabajo cooperativo, elaboración en grupo de un trabajo de investigación con presentación ante la clase, y un sistema de evaluación con procedimientos formativos que devolvían feedback a los estudiantes (dos entregas de portafolios, evaluación de presentaciones orales, rúbricas, coevaluación, autoevaluación del alumno, y pruebas escritas). Se encontraron mejoras estadísticamente significativas en las puntuaciones de enfoque profundo de aprendizaje, tanto en la puntuación global, de enfoque, como en las parciales de las dos dimensiones que evalúa el cuestionario, motivos y estrategias. No se encontraron diferencias estadísticamente significativas en las puntuaciones del enfoque superficial, aunque éstas disminuyeron.
\end{abstract}

Palabras clave: métodos innovadores, enseñanza centrada en el aprendizaje, enfoques de aprendizaje, alumnos universitarios

\title{
1. Introducción
}

En las dos últimas décadas se han ido estableciendo, a nivel teórico, en la universidad dos grandes modelos en el modo de explicar la relación entre los procesos de enseñanza y aprendizaje y también en el modo de abordar estos procesos, el modelo centrado en la enseñanza, centrado en el profesor, y el modelo centrado en el aprendizaje/centrado en el estudiante, de facilitación del aprendizaje (Barr y Tagg, 1995; Biggs, 2005; EI, ESU, 2010: García Valcárcel, 1993; Kember, 2009; Kember y Gow, 1994; Kember y Kwan, 2000; Monereo y Pozo, 2003; Samuelowicz y Bain, 2001 y 2002). El primer modelo pone énfasis en la transmisión de los conocimientos, en la instrucción, y concede especial relevancia a la figura del profesor, que es el depositario del conocimiento que tiene que transmitir a los estudiantes. El modelo centrado en el aprendizaje establece como prioritario el aprendizaje del alumno, el desarrollo de habilidades para el aprendizaje autónomo, la construcción del conocimiento en colaboración con los colegas y con el profesor. El papel del profesor también es relevante en este modelo y le exige actuar como mediador, como diseñador de entornos de aprendizaje que propicien el aprendizaje autónomo de los alumnos, lo que exige competencias pedagógicas, frente a modelos tradicionales centrados en el dominio de los contenidos y en su exposición ante los alumnos.

(c)) EY-NC-ND 2015, Universitat Politècnica de València 
Este modelo es el preconizado por el proceso de convergencia de Bolonia, con el que estamos comprometidos muchos países europeos, de cara a conseguir una formación de mayor calidad en nuestros estudiantes universitarios, y presenta como elementos relevantes la enseñanza innovadora -utilizando diferentes métodos propiciadores del aprendizaje activo: trabajo cooperativo, aprendizaje basado en problemas, desarrollo de proyectos, enseñanza de aprendizaje autorregulado, etc. compatibles con la metodología expositiva de calidad (Zabalza, 2012)-; el uso de una evaluación significativa, planteada como oportunidad de aprendizaje, utilizando diferentes fuentes de recogida de información, que devuelve feedback a los alumnos (Hernández, 2012) y les da la oportunidad de participar en el proceso -por ejemplo eligiendo vías y productos para mostrar los aprendizajes adquiridos-, y que fomenta también el aprendizaje del propio proceso de autoevaluación del estudiante (Hannafin, 2012); etc.

El desarrollo de este modelo exige un cambio en el papel del profesor y también en el papel del alumno, que no puede limitarse a ser el receptor y reproductor de los conocimientos transmitidos por el profesor, sino un sujeto activamente implicado en el proceso de aprendizaje, que debe indagar, cuestionar, elaborar, investigar, realizar aportaciones personales, que ha de participar activamente para convertir en significativo su proceso de aprendizaje (Machemer y Crawford, 2007), siendo capaz de conducirlo, estableciendo sus propias rutas de aprendizaje, autoevaluándose y autorregulándose (Hannafin, 2012).

En esta comunicación discutiremos el impacto de metodologías innovadoras/centradas en el aprendizaje, implementadas por dos profesores de la Universidad de Valencia que enseñan en el grado de Pedagogía, en los enfoques de aprendizaje de sus alumnos.

Los enfoques de aprendizaje son consistencias que hacen referencia a la manera como los estudiantes se enfrentan a una tarea académica, y se derivan tanto de las percepciones que el estudiante tiene de la tarea como de sus características personales (Biggs, 1993; Entwistle y Peterson, 2004). Los enfoques de aprendizaje se basan en motivos y utilizan ciertas estrategias (McCune y Entwistle, 2011). La tipología que suscribimos es la que postula que existen dos enfoques: profundo y superficial (Biggs, 1993; Entwistle, 1995).

La relevancia de la cuestión deriva del impacto que los enfoques de aprendizaje tienen en el rendimiento académico. Hay datos de ello de diferentes trabajos de investigación en distintos países: Valle et al. (2000), Biggs (1987), Zeegers (2001), Muñoz y Gómez (2005), Gargallo, Garfella y Pérez. (2006), De la Fuente, Pichardo, Justicia y Berbén (2008), y Bliuc, Ellis, Goodyear y Muntele (2011), entre otros.

El papel que desempeñan los estudiantes durante el proceso de enseñanza-aprendizaje puede ser modificado mediante planteamientos metodológicos centrados en el aprendizaje e innovadores (Gargallo, 2008). De hecho, los profesores siempre han tenido la convicción de que sus alumnos aprenden de una manera determinada en función de la forma en que se les enseña y evalúa. 


\section{Objetivos}

El objetivo que se persigue en este trabajo es verificar si la aplicación de métodos innovadores, centrados en el aprendizaje, desarrollados por profesores universitarios mejora los enfoques de aprendizaje de los estudiantes de la muestra seleccionada.

\section{Metodología de investigación}

\subsection{Diseño}

Se ha llevado a cabo un diseño preexperimental pretest-postest aplicados sobre dos grupos de alumnos, que cursaban la misma materia con distintos profesores, que utilizaron la misma metodología, centrada en el aprendizaje, en ambos grupos. No se pudo hacer uso de un diseño cuasiexperimental, que hubiera sido más sólido, dado que los profesores tradicionales, que continúan existiendo, no permiten que se recojan datos de sus estudiantes ni colaboran en este tipo de investigaciones.

\subsection{Participantes}

La muestra estuvo constituida por 112 estudiantes de primero de la Universidad de Valencia que cursaban Teoría de la Educación en $1^{\circ}$ curso del grados de Pedagogía (dos grupos, uno de ellos con 50 alumnos y el otro con 62). En cada uno de los grupos la materia era impartida por un profesor diferente.

El tipo de muestreo fue intencional, pues los participantes de la muestra fueron seleccionados a partir de una muestra de profesores que aplicaban métodos innovadores centrados en el aprendizaje en los grupos a los que pertenecían los estudiantes.

\subsection{Instrumentos}

Los enfoques de aprendizaje fueron evaluados por medio del cuestionario CPE (Cuestionario de Procesos de Estudio), versión española del R-SPQ-2 (Biggs, Kember y Leung, 2001). Es un cuestionario que consta de 20 ítems, divididos en dos escalas, una que evalúa el enfoque superficial y la otra el enfoque profundo, con diez ítems cada una. Las dos escalas están subdivididas en dos subescalas que evalúan motivos y estrategias (superficiales en una escala y profundos en la otra). El cuestionario emplea una escala de evaluación tipo Likert con cinco categorías que van desde "nunca o muy raramente" (1 punto) o "siempre o casi siempre" (5 puntos). La fiabilidad de las cuatro subescalas fue: motivo superficial, $\alpha=0.66$; estrategia superficial, $\alpha=0.67$; motivo profundo,$\alpha=0.61$; estrategia profunda, $\alpha=0.71$. La fiabilidad de las dos escalas fue: enfoque superficial, $\alpha=0.80$; enfoque profundo, $\alpha=0$.76.

\subsection{Procedimientos}

Los alumnos contestaron el cuestionario CPE al inicio de la docencia de la materia (pretest), contextualizando sus respuestas en su modo habitual de aprender, y los volvieron 
a contestar a su final (postest) contextualizando las respuestas en los profesores y en la materia que estaban cursando, mediante la web https://poliformat.upv.es/portal.

Los resultados fueron procesados con el paquete estadístico SPSS 19.0. Se realizó la prueba $t$ de Student de diferencia de significación de medias para analizar las posibles diferencias existentes en las puntuaciones de enfoques de aprendizaje entre pretest y postest.

\section{Desarrollo de la innovación}

Los tres profesores consensuaron la metodología a utilizar en sus clases, que se pretendía fuera innovadora/centrada en el aprendizaje. Tal metodología hacía uso, para la enseñanza, de los siguientes métodos:

- lección magistral participativa

- planteamiento de preguntas a responder por los alumnos antes de la clase por el aula virtual con trabajo autónomo de materiales

- discusión en pequeño grupo sobre las cuestiones elaboradas, puesta en común en gran grupo y discusión general con la mediación del profesor.

- realización de prácticas de aula sobre los contenidos trabajados, que incluían aplicaciones prácticas, estudio de casos, simulaciones, vídeos, uso de técnicas pedagógicas, etc.

- trabajo cooperativo para la realización de las prácticas en clase

- elaboración de un trabajo de investigación realizado mediante trabajo cooperativo, que incluía trabajo de campo para la recogida de datos, sesiones presenciales en el aula supervisadas y asesoradas por los profesores mediante seminarios grupales, y exposición y defensa en gran grupo de clase con coevaluación

- realización de un portafolios que incluía las cuestiones trabajadas en clase, las actividades prácticas desarrolladas, un repertorio de cuestiones para la reflexión metacognitiva sobre el trabajo realizado, las dificultades y las alternativas desarrolladas, y también autoevaluación.

Para la evaluación se utilizaron diversas herramientas:

- portafolios (incluía dos entregas corregidas por los profesores con devolución a los alumnos para ofertar feed-back de cara a ayudarles a la mejora del proceso de aprendizaje) (su valor era 60\% de la calificación final, correspondiendo 20\% a las cuestiones elaboradas por los alumnos, 20\% a los informes de prácticas y $20 \%$ al trabajo de investigación realizado en grupo)

- prueba escrita final de respuesta abierta sobre los contenidos trabajados (su valor era el $40 \%$ de la calificación final)

- coevaluación de la presentación del trabajo de investigación por parte de los alumnos, a partir de una rúbrica que incluía los criterios de evaluación

- autoevaluación del propio estudiante 
Los alumnos disponían de rúbricas publicadas por su profesor para la evaluación de las dos entregas del portafolios, y también para llevar a cabo la coevaluación de la presentación oral de trabajos, con el objetivo de que fueran conscientes de los criterios de evaluación y de que desarrollaran progresivamente habilidades más finas de autoevaluación.

\section{Resultados}

Los resultados obtenidos se recogen en la tabla 1 . Se encontraron mejoras estadísticamente significativas en las puntuaciones de enfoque profundo de aprendizaje, tanto en la puntuación global, de enfoque ( $\mathrm{p}<.01)$, como en las parciales de las dos dimensiones que evalúa el cuestionario, motivos $(\mathrm{p}<.05)$ y estrategias $(\mathrm{p}<.01)$. Las puntuaciones de enfoque profundo se incrementaron en el postest.

No se encontraron diferencias estadísticamente significativas en las puntuaciones del enfoque superficial, aunque éstas disminuyeron en el postest.

Tabla 1. Resultados de la prueba t de Student en enfoques de aprendizaje pretest-postest

\begin{tabular}{|c|c|c|c|c|c|c|c|}
\hline & & Media & Desviación Tip. & $\mathrm{N}$ & $\overline{G L}$ & $\mathrm{t}$ & Signif. \\
\hline \multirow[t]{2}{*}{ Par 1} & Motivo superficial_pre & 1,9478 & ,61099 & 112 & 111 & 804 & ,423 \\
\hline & Motivo superficial_post & 1,9071 & ,55079 & & & & \\
\hline \multirow[t]{2}{*}{ Par 2} & Estrategia superficial_pre & 2,3719 & ,66396 & & &,- 007 & 994 \\
\hline & Estrategia superficial_post & 2,3723 & 65185 & & & & \\
\hline \multirow[t]{2}{*}{ Par 3} & Estrategia profunda_pre & 3,1179 & 67999 & & & $-2,997$ & ,003 \\
\hline & Estrategia profunda_post & 3,3000 & 63901 & & & & \\
\hline \multirow[t]{2}{*}{ Par 4} & Motivo profundo_pre & 3,3920 & 67278 & & & $-2,156$ & ,033 \\
\hline & Motivo profundo_post & 3,5214 & 66001 & & & & \\
\hline \multirow[t]{2}{*}{ Par 5} & Enfoque superficial_pre & 2,1588 & ,57933 & & & ,388 & ,699 \\
\hline & Enfoque superficial_post & 2,1397 & ,56173 & & & & \\
\hline \multirow[t]{2}{*}{ Par 6} & Enfoque profundo_pre & 3,2549 & 61880 & & & $-2,988$ & ,003 \\
\hline & Enfoque profundo_post & 3,4100 & 60722 & & & & \\
\hline
\end{tabular}

\section{Conclusiones}

El objetivo perseguido en este trabajo era verificar si la aplicación de métodos innovadores, centrados en el aprendizaje, desarrollados por profesores universitarios, mejoraba los enfoques de aprendizaje de los estudiantes de la muestra seleccionada.

Los resultados obtenidos muestran que los métodos centrados en el aprendizaje mejoraron los enfoques de aprendizaje de los alumnos, que incrementan su enfoque profundo de aprendizaje y reducen el superficial. Ello es coherente con el trabajo que estos métodos exigen al estudiante, ya que los alumnos han de trabajar en profundidad los materiales para responder a las cuestiones que han de resolver en cada tema; por otra parte, el trabajo de discusión realizado en clase en pequeño grupo y en gran grupo, con la mediación del profesor, potencia la comprensión y el enfoque profundo de aprendizaje. Lo mismo ocurre con las prácticas de clase, el trabajo de investigación que se realiza a lo largo del cuatrimestre y las presentaciones públicas de sus trabajos. También ayuda el planteamiento 
de la evaluación, de tipo formativo, con las dos entregas del portafolios y las sucesivas revisiones que se pueden efectuar a partir de la corrección del profesor.

Somos conscientes de que lo ideal sería implementar métodos centradas en el aprendizaje en titulaciones y centros completos y, si fuera posible, en toda la universidad (Kember, 2009). Sabemos de los obstáculos que hay que salvar para ello: necesidad de cambios organizativos (De La Sablonière et al., 2009), formación de calidad para los profesores, aspectos motivacionales de profesores y alumnos (Maclellan, 2008), etc. Mientras tanto, trabajos como el desarrollado por profesorado comprometido pueden servir de acicate para que otros profesores se incorporen a esta dinámica.

\section{Referencias}

BARR, R.B. y TAGG, J. (1995). "From teaching to learning. A new paradigm for undergraduate education”, en Change, 27 (6), pp. 13-25.

BIGGS, J. (1987). Students Approaches to Learning and Studying. Melbourne: Council for Educational Research.

BIGGS, J. (1993). "What do inventories of students' learning processes really measure? A theoretical review and clarification", en British Journal of Educational Psychology, 63, 3-19.

BIGGs, J. (2005). Calidad del aprendizaje universitario. Madrid: Narcea.

Biggs, J., KemBer, D. y Leung, D.Y.P. (2001). "The revised two-factor Study Process Questionnaire: R-SPQ-2”, en British Journal of Educational Psychology, 71, 133-149.

Bliuc, A-M., ElLis, R.A., GoOdyeAR, P. y MunTele, D. (2011). "The role of social identification as university student in learning: relationships between students' social identity, approaches to learning, and academic achievement”, en Educational Psychology, 31 (5), 559-574.

DE LA SABLONNIÈRE, R., TAYLOR, D. M. y SADYKOVA, N. (2009). "Challenges of applying a studentcentred approach to learning in the context of Education in Kyrgystan”, en International Journal of Educational Development, 29, 628-634.

Entwistle, N. (1995). "Frameworks for understanding as experienced in essay writing and in preparing for examinations”, en Educational Psychologist, 30, 47-54.

ENTwistLe, N. y Peterson, E. (2004). "Learning styles and approaches to studying”, en Spielberger, Ch. (Ed.). Encyclopedia of Applied Psychology, Vol 2 (pp. 537-542). Amsterdam: Elsevier.

De la Fuente, J., Pichardo, M. C., Justicia, F., y Berbén, A. (2008). "Enfoques de aprendizaje, autorregulación y rendimiento en tres universidades europeas”, en Psicothema, 20(4), 705-711.

García ValCÁrCel, A. (1993). "Análisis de los modelos de enseñanza empleados en el ámbito universitario”, en Revista Española de Pedagogía, 194, 27-53.

MUÑoz, E. y GÓMEZ, J. (2005). “Enfoques de aprendizaje y rendimiento académico de los estudiantes universitarios”, en Revista de Investigación Educativa, 23 (2), 417-432.

GaRgallo, B. (2008): "Estilos de docencia y evaluación de los profesores universitarios y su influencia sobre los modos de aprender de sus estudiantes”, en Revista Española de Pedagogía, 241, 425-445.

Hannafin, M. (2012). "Student-Centered Learning”, en Seel, N.M. (Ed.), Encyclopedia of the Sciences of Learning (pp. 3211-3214). Nueva York: Springer. Recuperado de http://link.springer.com/content/pdf/bfm\%3A978-1-4419-1428-6\%2F1.pdf

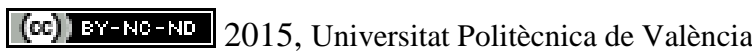

Congreso In-Red (2015) 
Métodos innovadores y enfoques de aprendizaje en estudiantes universitarios

HERNÁNDEZ, R. (2012). “Does continuous assessment in higher education support student learning?”, en Higher Education, 64, 489-502. DOI 10.1007/s10734-012-9506-7

Kember, D. (2009). "Promoting student-centred forms of learning across an entire university”, en Higher Education, 58, 1-13.

KEMBER, D. y Gow, L. (1994). “ Orientations to teaching and their effects on the quality of student learning”, en Journal of Higher Education, 65 (1), 59-74.

KEMBER, D. y KWAN, K. (2000). "Lecturers' approaches to teaching and their relationship to conceptions of good teaching”, en Instructional Science, 28, 469-490.

MACHEMER, P.L. y CRAWFORD, P. (2007). "Student perceptions of active learning in a large crossdisciplinary classroom”, en Active Learning in Higher Education, 8 (1), 9-30.

MACLELLAN, E. (2008). "The significance of motivation in student-centred learning: a reflective casestudy”, en Teaching in Higher Education, 13 (4), 411-421.

McCune, V. y EnTwistle, N. (2011). "Cultivating the disposition to understand in $21^{\text {st }}$ century university education”, en Learning and Individual Differences, 21 (3), 303-310.

Monereo, C. y Pozo, J. I. (2003). La universidad ante la nueva cultura educativa. Enseñar y aprender para la autonomía. Madrid: Síntesis.

MuÑoz, E. y GómEz, J. (2005). "Enfoques de aprendizaje y rendimiento académico de los estudiantes universitarios”, en Revista de Investigación Educativa, 23 (2), 417-432.

SAMUELOWICZ, K. y BAIN, J.D. (2001). "Revisiting academics' beliefs about teaching and learning”, en Higher Education, 41, 299-325.

SAMUELOWICZ, K. y BAIN, J.D. (2002). “ Identifying academics’ orientations to assessment practice”, en Higher Education, 43, pp. 173-201.

Zabalza, M.A. (2012). “Metodología docente”, en REDU (Revista de Docencia Universitaria, 9 (3), 75-98.

ZEEgERs, P. (2001). “Approaches to learning in science: a longitudinal study”, en British Journal of Educational Psychology, 71, 115-132. 